\title{
ORIGINAL ARTICLE \\ Proton magnetic resonance spectroscopy of human cervical spondylosis at 3T
}

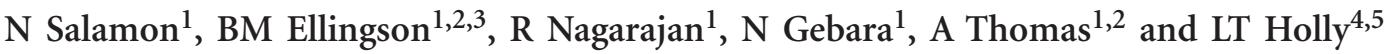

\begin{abstract}
Study design: A single-center magnetic resonance imaging and spectroscopic study involving 21 patients with advanced cervical spondylosis and 11 healthy controls.

Objective: We assessed the utility of magnetic resonance spectroscopy (MRS) to quantify biochemical changes within the spinal cord and serve as a potential biomarker in patients with cervical spondylosis with or without T2 hyperintensity within the cord.

Setting: Los Angeles, California, USA.

Methods: Twenty-one patients with cervical spondylosis and eleven healthy controls were evaluated. Single-voxel MRS was performed in the cervical cord. Morphometry of the spinal canal space was measured. N-Acetyl aspartylglutamic acid (NAA), choline (Cho), myo-inositol (Myo-I), glutamine-glutamate complex (Glx) and lactate metabolite concentration ratios with respect to total creatine (Cr) were quantified using an LC model algorithm and compared between healthy controls and spondylosis patients. Correlation of MRS metabolites with modified Japanese Orthopaedic Association (mJOA) score was also performed.

Results: The spinal canal space was significantly different between patients and controls (analysis of variance (ANOVA), $P<0.0001$ ). Total Cho-to-Cr ratio was significantly elevated in patients with spondylosis and T2-hyperintensity compared with healthy controls (ANOVA, $P<0.01$ ). A significantly higher Cho-to-NAA ratio was observed in spondylosis patients compared with healthy controls (ANOVA, $P<0.01$ ). Slightly elevated Glx and Myo-I were encountered in patients with stenosis without T2 hyperintensity. A linear correlation between Cho-NAA ratio and mJOA was also observed $(P<0.01)$.

Conclusion: MRS appears sensitive to biochemical changes occurring in advanced cervical spondylosis patients. The Cho/NAA ratio was significantly correlated with the mJOA score, providing a potentially clinically useful radiographical biomarker for the management of advanced cervical spondylosis patients.
\end{abstract}

Sponsorship: NIH NINDS 1R21NS65419-1A1; NIH/NINDS 1R01NS078494-01A1.

Spinal Cord (2013) 51, 558-563; doi:10.1038/sc.2013.31; published online 16 April 2013

Keywords: MR spectroscopy; spinal cord; cervical spondylosis; stenosis; MRI; myelopathy

\section{INTRODUCTION}

Cervical spondylotic myelopathy (CSM), is the most common cause of nontraumatic spastic paresis, ${ }^{1}$ the most common cause of spinal dysfunction in the elderly, ${ }^{2}$ and it is the most common primary diagnosis for patients over 64 years who undergo cervical spine surgery. ${ }^{3}$ In order to prevent neurological deterioration in patients with symptomatic cervical spondylosis, surgery is often performed early after diagnosis. Some studies have suggested surgical intervention after significant neurological decline in these patients may not result in neurological improvement; ${ }^{4}$ therefore, surgical intervention may be considered in some cases on the basis of diagnostic imaging even in the absence of overt clinical symptoms. ${ }^{5}$ The value of traditional imaging technologies in diagnosing and predicting response to treatment, however, remains controversial.

Magnetic resonance imaging (MRI) is currently the gold standard for the assessment of the cervical spinal cord. As such, many studies have attempted to investigate the relationship between pathophysiological changes found on MRI and the patient clinical condition. Although abnormal signal on routine T1- and T2-weighted MRI are correlated with morphological changes and cavitation in experimental canine models of compressive myelopathy, ${ }^{6}$ and with edema and gliosis in humans, ${ }^{7}$ clinical studies have not shown consistent correlation between T1- and T2-weighted MRI findings and patient clinical status, or outcome after either surgical or medical treatment. ${ }^{8,9}$

Preliminary studies have suggested magnetic resonance spectroscopy (MRS) may be useful for quantitatively assessing subtle biochemical changes within the spinal cord that may precede morphologic changes observed with traditional imaging techniques. ${ }^{10-12}$ Our, previous investigations using a $1.5-\mathrm{T}$ MR scanner confirmed the feasibility of accurately performing MRS in symptomatic CSM patients. ${ }^{10}$ In the current study, we explore the use of quantitative MRS in the cervical spinal cord in healthy control participants and a spectrum of patients with cervical spondylosis using a 3-T MR scanner. We hypothesize MRS may be a useful technique for evaluating spinal cord biochemistry in both

${ }^{1}$ Department of Radiological Sciences, David Geffen School of Medicine, University of California Los Angeles, Los Angeles, CA, USA; ${ }^{2}$ Department of Biomedical Physics, David Geffen School of Medicine, University of California Los Angeles, Los Angeles, CA, USA; ${ }^{3}$ Department of Bioengineering, Henry Samueli School of Engineering and Applied Science, University of California Los Angeles, Los Angeles, CA, USA; ${ }^{2}$ Department of Neurosurgery, David Geffen School of Medicine, University of California Los Angeles, Los Angeles, CA, USA and ${ }^{5}$ Department of Orthopaedics, David Geffen School of Medicine, University of California Los Angeles, Los Angeles, CA, USA

Correspondence: Dr LT Holly, Department of Neurosurgery, David Geffen UCLA School of Medicine, University of California Los Angeles, 10833 Leconte Avenue, Los Angeles, CA 90095, USA.

E-mail: Iholly@mednet.ucla.edu

Received 1 March 2013; accepted 18 March 2013; published online 16 April 2013 
symptomatic and asymptomatic patients, and help to elucidate pertinent information about cellular metabolic function not provided by conventional MRI.

\section{MATERIALS AND METHODS}

\section{Patient population}

The study group was composed of 21 patients who were evaluated at a Neurosurgery clinic for signs and symptoms related to cervical spondylosis. There were 12 female and 9 male patients, and the mean age was 59 years. Baseline history and physical examinations were performed on each patient. Conventional MRI demonstrated advanced cervical spondylosis, with at least moderate central spinal canal stenosis in each case. Ten of the patients had increased T2 weighted signal within the spinal cord (Figure 1a), and the other 11 had normal signal intensity throughout the spinal cord (Figure 1b).

Exclusion criteria included (1) spinal cord compression at the C2 level or cervicomedullary junction, (2) previous cervical spine surgery, (3) acute change in neurological function related to central cord syndrome or other traumatic event, (4) cardiac pacemaker or other non-MRI compatible implant, (5) severe claustrophobia. Eleven healthy volunteers without evidence of neurological symptomatology underwent cervical spine MRS using the same protocol and served as the control group. The Office for the Protection of Research Subjects at our Institution approved the protocol for this study.

\section{Clinical presentation}

Motor dysfunction was the most commonly encountered symptom in the stenosis with signal change cohort, as five of the patients presented with gait/ and or hand incoordination. Of the remaining patients in the group, four presented with a primary complaint of neck pain, and two with paresthesias in the upper extremities.

In the cervical spondylosis cohort without $\mathrm{T} 2$ signal change, four patients presented with a primary complaint of neck pain. An additional four patients
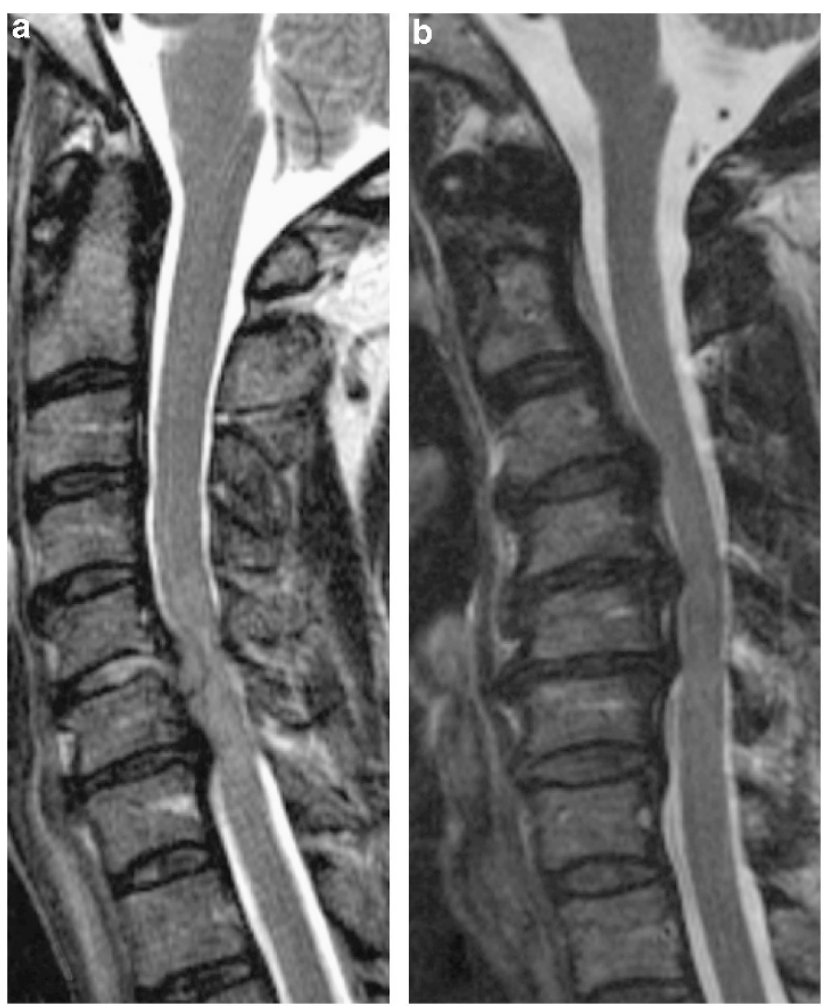

Figure 1 (a) T2 weighted sagittal MRI of a 68 -year-old gentleman with significant spinal cord compression and signal change. (b) T2 weighted sagittal MRI of a 56-year-old patient with significant spinal cord compression without signal change. noted paresthesias in the upper extremity as their primary symptom. One patient had complained of gait/hand dysfunction, and the remaining patient was asymptomatic.

\section{Clinical functional assessment}

The Japanese Orthopaedic Association (JOA) score is the most commonly used functional outcome assessment instrument in CSM patients, and has been demonstrated to be a valid and reliable outcome measurement in this population. ${ }^{11}$ This study uses the modified JOA (mJOA) scale, a slightly modified version that accounts for differences in western culture. ${ }^{12}$ The scale ranges from 3 to 18 points, and evaluates motor and sensory function in the extremities as well as sphincter function. Ascending point values represent better neurological function.

\section{Proton MRS}

All patients and control underwent cervical imaging using a same Trio Tim 3.0T Siemens system (Siemens Medical Solution, Erlangen, Germany) running on a vb17 platform. The subjects underwent conventional T2 sagittal (repetition time $(\mathrm{TR}) / \mathrm{echo}$ time $(\mathrm{TE})=4000 / 103 \mathrm{~ms}), \mathrm{T} 2$ axial $(\mathrm{TR} / \mathrm{TE}=3500 /$ $82 \mathrm{~ms}$ ) and coronal HASTE images. Single-voxel MRS was obtained with point resolved spectroscopy sequence with water suppression: $\mathrm{TR} / \mathrm{TE}=2000 \mathrm{~ms} /$ $30 \mathrm{~ms}, 256$ Averages, voxel size $1.72 \mathrm{ml}\left(7 \times 7 \times 35 \mathrm{~mm}^{3}\right)$. Water-enhanced T1 saturation effects were applied before the selected localization technique. Three frequency selective pulses are applied along with a dephasing gradient to suppress the water. A non-water suppressed spectrum with four averages was also obtained for eddy current correction. The voxel was placed at the posterior portion of the C2 vertebral body, using sagittal T2 weighted images. For the outer volume saturations, six bands were applied for presaturation of fat containing regions on each side of the voxel. Manual shimming was performed to calculate optimal shim currents. All MRS metabolites were quantified by fitting experimental data in the frequency domain using the LC model algorithm. ${ }^{13,14}$ Ratios of the following metabolite concentrations were calculated with respect to total creatine $(\mathrm{Cr})$ concentration (that is, $\mathrm{Cr}$ plus phosphocreatine): $\mathrm{N}$-acetyl-aspartate and $\mathrm{N}$-acetyl aspartylglutamic acid (NAA), glutamine and glutamate complex (Glx), glycerophosphorylcholine and phosphocholine (Cho) and myo-inositol (Myo-I; Table 1).

\section{Anterior-posterior (A-P) canal space}

A-P canal space, also referred to as the 'space available for the cord', reflects the total A-P distance between the spinal cord and vertebral body. This value was calculated by measuring the A-P diameter of the spinal canal, then subtracting the measured A-P diameter of the spinal cord on midline sagittal T2 weighted images at each intervertebral level in the cervical spine. This technique has been used extensively as a measure of spinal cord compression. ${ }^{15-17}$

Table 1 Spinal cord metabolite concentrations in normal control subjects, cervical stenosis patients without T2 signal changes and cervical stenosis patients with T2 signal changes within the spinal cord

\begin{tabular}{|c|c|c|c|}
\hline & $\begin{array}{l}\text { Normal control } \\
\quad(n=11)\end{array}$ & $\begin{array}{c}\text { Cervical stenosis } \\
\text { (No T2 changes; } \\
\quad \mathrm{n}=11 \text { ) }\end{array}$ & $\begin{array}{c}\text { Cervical stenosis } \\
\text { (T2 signal changes; } \\
\mathrm{n}=10 \text { ) }\end{array}$ \\
\hline $\mathrm{NAA} / \mathrm{Cr}$ & $1.373 \pm 0.322^{\mathrm{a}}$ & $1.267 \pm 0.269$ & $1.174 \pm 0.422$ \\
\hline $\mathrm{Cho} / \mathrm{Cr}$ & $0.309 \pm 0.083$ & $0.405 \pm 0.088$ & $0.486 \pm 0.167$ \\
\hline $\mathrm{Glx} / \mathrm{Cr}$ & $1.676 \pm 0.998$ & $2.024 \pm 1.440$ & $1.356 \pm 0.780$ \\
\hline $\begin{array}{l}\text { (Lip/Lac)/ } \\
\mathrm{Cr}\end{array}$ & $0.189 \pm 0.133$ & $0.274 \pm 0.209$ & $0.356 \pm 0.383$ \\
\hline Myo- $/ / \mathrm{Cr}$ & $1.422 \pm 0.573$ & $1.488 \pm 0.575$ & $1.309 \pm 0.672$ \\
\hline Cho/NAA & $0.245 \pm 0.083$ & $0.322 \pm 0.049$ & $0.344 \pm 0.069$ \\
\hline
\end{tabular}

Abbreviations: Cho, choline; $\mathrm{Cr}$, creatine; Glx, glutamine and glutamate complex; Lip, lipid; Lac, lactate; Myo-I, myo-inositol; NAA, N-acetyl aspartylglutamic acid. a Error measurements represent standard deviation. 


\section{Statement of ethics}

We certify that all applicable institutional and governmental regulations concerning the ethical use of human volunteers were followed during the course of this research.

\section{Statistical analysis}

SPSS (Statistical Package for Social Sciences, SPSS Inc, Chicago, IL, USA) version 15.0 and GraphPad Prism version 4.0 (GraphPad Software, Inc, La Jolla, CA, USA) were used for statistical analyses. Comparisons between the groups were performed using an ANOVA. Pearson's correlation coefficient was used for correlation analysis. A P-value of less than 0.05 was considered to be significant.

\section{RESULTS}

Morphometric analysis of standard MR images demonstrated significantly greater compression of the spinal cord (lower A-P spinal cord space) in both groups of spondylosis patients compared with healthy control participants (Figure 2; two-way ANOVA, $P<0.0001$ ). There was no significant difference between the A-P spinal cord space between cervical stenosis patients with T2 signal change and those without spinal cord signal change. The mean mJOA score was not significantly different in cervical stenosis patients with T2 signal change compared with patients lacking observable T2 signal change within the cord, although patients with $\mathrm{T} 2$ hyperintensity trended toward lower mJOA scores (14.7 vs $16.6, t$-test, $P=0.0984$ ).

Quantitative MRS of the cervical spinal cord was successfully obtained in 11 healthy controls (Figure 3) and all 21 cervical spondylosis patients (Figure 4). Implementation of short TE $(\mathrm{TE}=30 \mathrm{~ms})$ in point resolved spectroscopy sequence, MRS acquisition allowed for clear identification of NAA, Cr, Cho, lactate, lipids and Myo-I peaks along with quantification of concentrations using the LC model algorithm fitted to experimental data in the frequency domain. The relative concentration of total Cho to total $\mathrm{Cr}(\mathrm{Cho} / \mathrm{Cr})$ was significantly lower in healthy controls $(0.309 \pm 0.083)$ than cervical stenosis patients with T2 signal change within the cord $(0.486 \pm 0.167$; one-way ANOVA, $P=0.0065$; Tukey's test for multiple comparisons, control vs cervical stenosis with T2 changes, $P<0.01$ ); however, no significant differences in $\mathrm{Cho} / \mathrm{Cr}$ were observed between healthy controls and patients with cervical stenosis without T2 signal changes within the spinal cord (Tukey's test, $P>0.05$; Figure $5 \mathrm{a}$ ).

We observed significantly lower Cho/NAA in healthy controls compared with both cervical stenosis patients with T2 signal changes (Figure 5b; Tukey's test, $P<0.01$ ) and those without appreciable T2 signal changes within the cord (Tukey's test, $P<0.05$ ). Linear regression between Cho/NAA and mJOA score verified this relationship, showing a correlation between a lower Cho/NAA and higher mJOA score (Pearson's correlation coefficient, $R=-0.45, P=0.013$ ).
Although the NAA/Cr was higher in control subjects than the cervical stenosis patients, this relationship did not reach significance. Both glutamine and myoinositol levels relative to $\mathrm{Cr}$ were highest in the stenosis without myelomalacia cohort, but these ratios were not significantly different between groups.

\section{DISCUSSION}

Degenerative disc disease occurs as part of the normal aging process and its treatment costs run in the many billions of dollars per year. CSM is the most debilitating form of degenerative disc disease, and is caused by progressive abnormalities of the vertebral column that result in spinal cord damage due to both primary mechanical and secondary biological injury. Not uncommonly, deterioration can progress from ambulating without assistance, to requiring a cane, to a walker and ultimately become wheelchair bound over a period of 6 months to a year from the onset of symptoms.

Although chronic spinal cord injury is a widely studied hallmark of CSM, the time course and evolution of the cellular and microstructural damage is poorly understood. This is in large part due to the fact that standard MRI provides excellent macroscopic anatomical detail, yet extremely limited specific information regarding the spinal cord cellular function and micro-architecture. We postulate that there is a spectrum of cellular and microstructural changes that occur within the spinal cord as patients with cervical spondylosis progress from being asymptomatic to manifesting neurological impairment. It is well established that surgical intervention before the onset of irreversible spinal cord injury is associated with better neurological outcomes. Thus, an understanding of the real-time spinal cord cellular alterations that occur as patients progress to a symptomatic state would be a significant advancement in the treatment of cervical spondylosis patients.

MRS is able to provide pertinent information regarding cellular biochemical function, and therefore may be able to provide novel insight into the cellular derangements associated with CSM. We previously studied the ability of MRS to identify axonal injury by measuring NAA/Cr levels in a series of symptomatic CSM patients using a 1.5 -T MRI scanner. ${ }^{10}$ In the present study, we evaluated not only symptomatic CSM patients, but also asymptomatic advanced cervical spondylosis patients with evidence of spinal canal stenosis, in order to obtain a broad sampling of clinical condition and radiographical findings. The cervical stenosis with and without signal change cohorts had a similar degree of spinal canal stenosis, which was significantly greater than the control group. The major objectives of this study were to examine the cellular changes that occur in patients with advanced cervical spondylosis, and to assess the feasibility of MRS to provide cellular biomarkers that could be utilized to discern neurological status in this patient population.
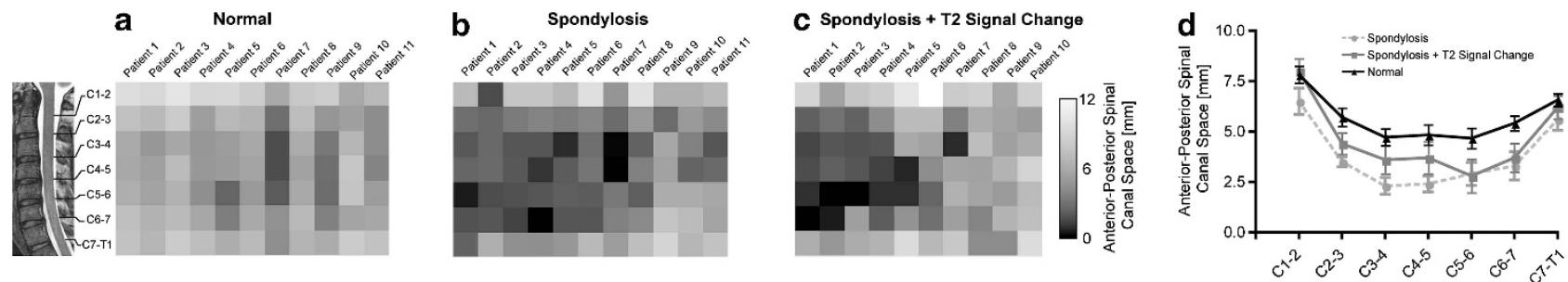

Figure 2 Anterior-posterior (A-P) spinal canal space in the cervical spine for healthy volunteers, cervical stenosis without signal change, and cervical stenosis patients with signal change. (a) Heat maps pictorially illustrating A-P space across the cervical spine in normal healthy volunteers. (b) Heat maps pictorially illustrating A-P space across the cervical spine in patients with cervical stenosis without signal change. (c) Heat maps illustrating A-P space across the cervical spine in patients with cervical stenosis and signal change. (d) Group average A-P spinal canal space across the cervical spine. Error bars represent standard error of the mean (s.e.m.). 


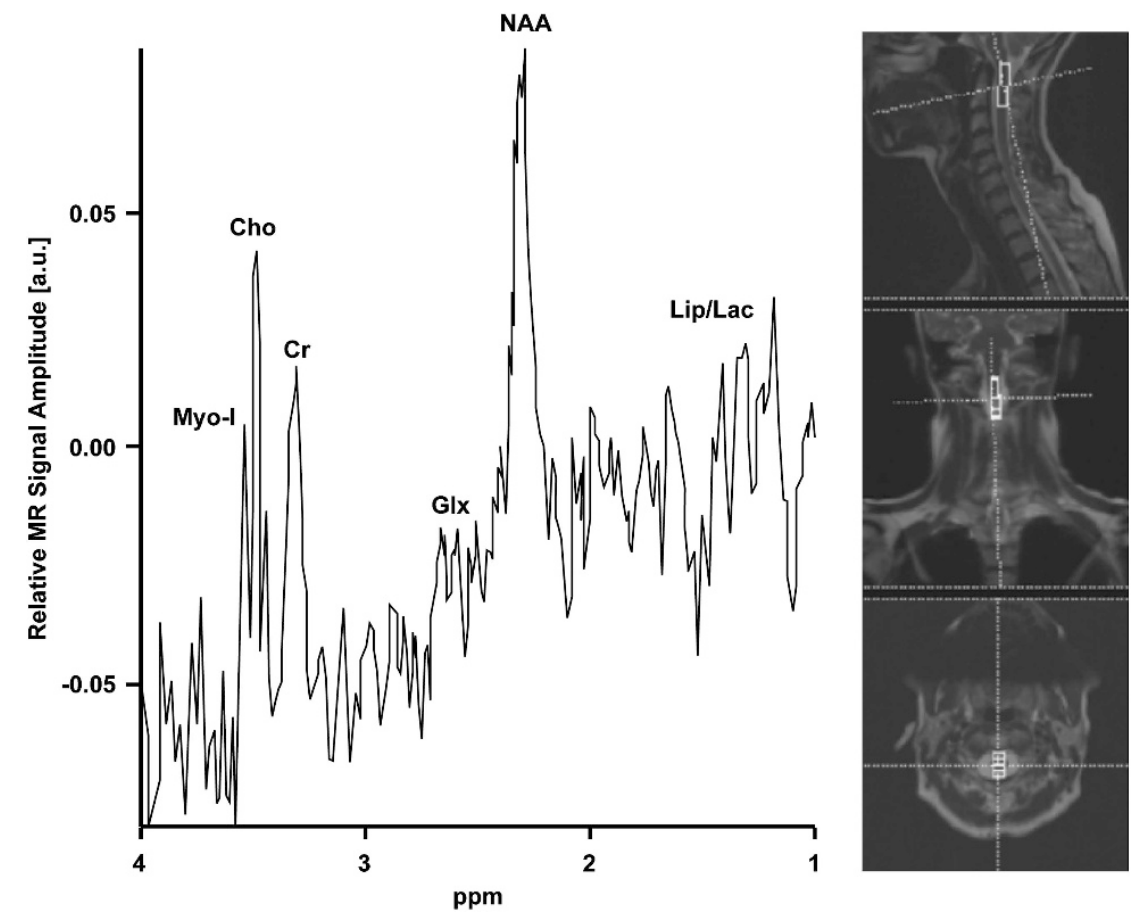

Figure $3{ }^{1} \mathrm{H}$-MR spectrum and voxel localization images of the upper cervical spinal cord in a 44-year-old healthy volunteer.

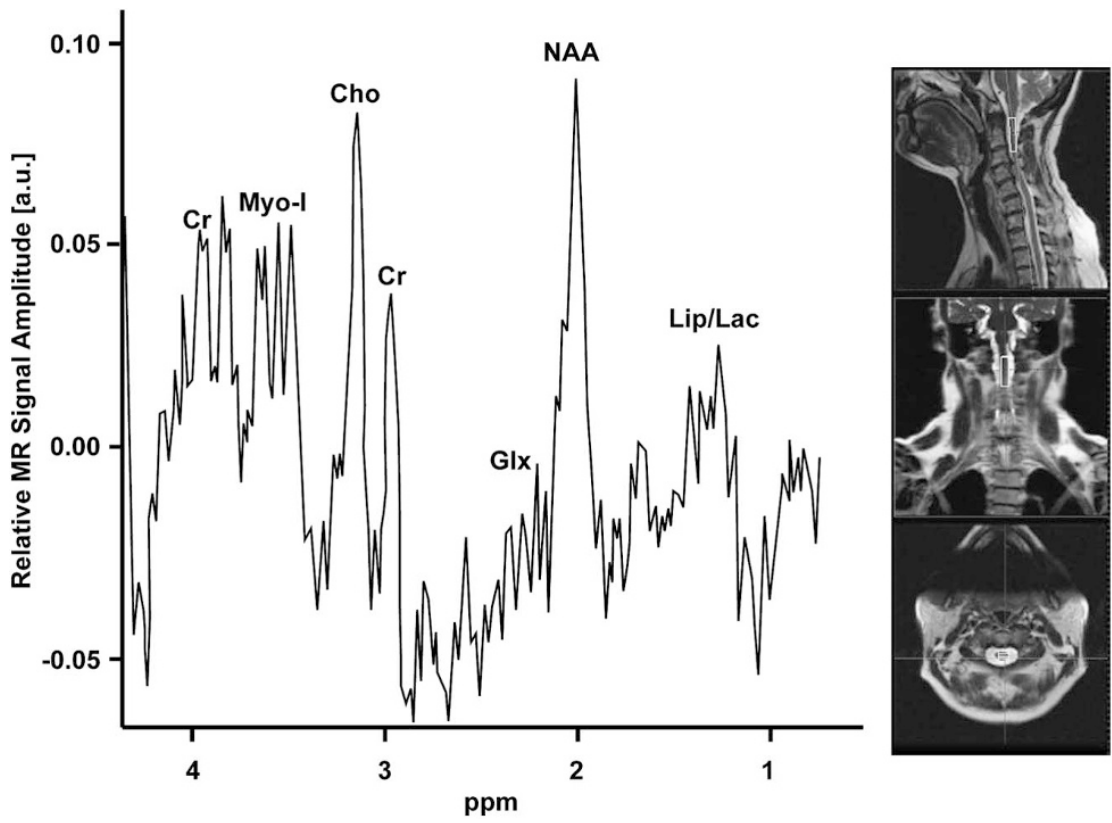

Figure $4{ }^{1} \mathrm{H}$-MR spectrum and voxel localization images of the upper cervical spinal cord in a 49 -year-old patient with cervical stenosis and myelomalacia.

Early changes in MRS metabolites

Patients with cervical stenosis without spinal cord signal change showed slightly higher Myo-I and glutamate-glutamine when compared with healthy controls. This is a relatively novel finding that has not been observed in previous MRS studies of patients with spinal traumatic or degenerative spinal cord lesions. ${ }^{10,18}$ Marginally increased Myo-I, however, has been detected in the spinal cord of patients with multiple sclerosis with acute ${ }^{19}$ and chronic lesions ${ }^{20}$ when compared with controls. An increased Myo-I suggests that reactive gliosis, including proliferation and astrocytic hypertrophy, may occur in the spinal cord rostral from the site of injury, possibly in response to the Wallerian degeneration of injured neuronal fibers. This is in agreement with an increase in vimentin and glial fibrillary acidic protein immunoreactivity, which is a marker of proliferation of astrocytes and gliosis, described in animal models of acute and chronic stage after spinal cord injury. ${ }^{21,22}$ This suggests Myo-I could 
a

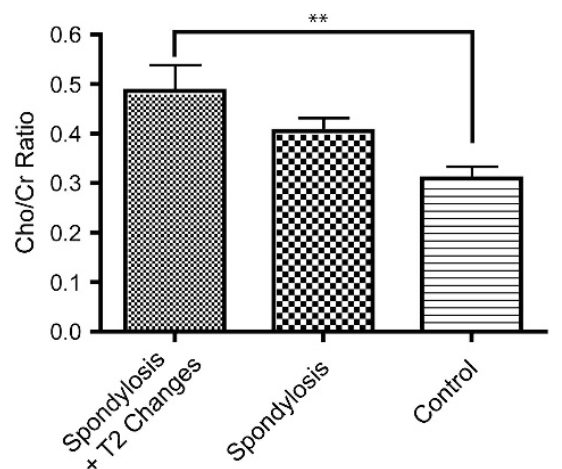

b

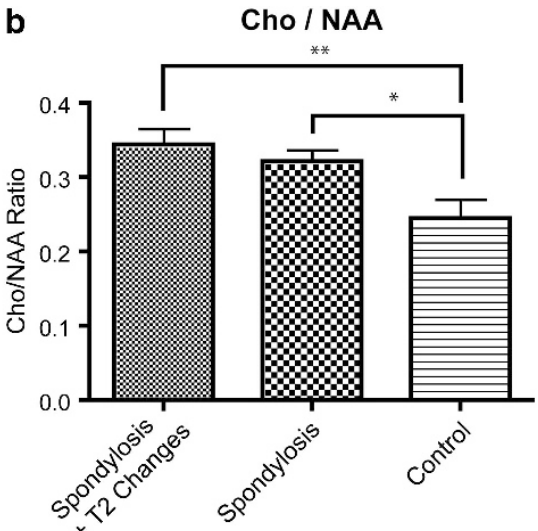

Figure 5 (a) Metabolite levels in control $(n=11)$, stenosis $(n=11)$ and stenosis with signal change $(n=10)$ patients. A significantly higher Cho/Cr was observed in spondylosis patients with signal change vs control subjects $(* * P<0.01)$. (b) Spondylosis patients (with or without signal change) showed a significantly higher Cho/NAA compared with control subjects (control vs spondylosis without signal change, * $P<0.05$; control vs spondylosis with signal change, ${ }^{* *} P<0.01$ )

potentially be an early marker for spinal cord inflammation and early stage of demyelination in cervical stenosis patients before neurological impairment.

Glx is an excitatory neurotransmitter and found in early neuronal damage. Elevated Glx has been reported in early traumatic brain injury, ${ }^{23}$ multiple sclerosis and early stage of spinal cord injury in animal study. ${ }^{22}$ For example, Erschbamer et al. ${ }^{22}$ demonstrated change in Glx in the rat spinal cord after traumatic injury. Together, the observation of slightly elevated Myo-I and Glx in cervical stenosis patients without spinal cord signal change suggests stenosis can precipitate an inflammatory process within the spinal cord, resulting in early demyelination and neurotransmitter metabolic dysfunction before myelopathy or neuronal axonal damage, which may then ultimately lead to neurological symptoms.

\section{Late changes in MRS metabolites}

The Cho/Cr ratio was significantly higher in stenosis patients with spinal cord signal change than the control cohort, however, was not significantly different between the stenosis patients without spinal cord signal change and the control group. This finding suggests that increased Cho levels may occur later than some of the other aforementioned cellular metabolic changes as cervical spondylosis progresses to a symptomatic state. The increased Cho/NAA ratio in cervical stenosis patients compared with healthy controls observed is consistent with previous MRS studies in other spinal disorders, such as chronic whiplash $(-, 18)$ and in patients with multiple sclerosis. ${ }^{21,24}$ Reduced NAA levels indicate both axonal loss and metabolic dysfunction, ${ }^{25,26}$ whereas increased total Cho levels suggest increased membrane turnover from degeneration. Previous investigations have shown a cascade of microstructural degenerative changes within the spinal cord after contusive injury, which propagates in a predictive fashion in both the rostral and cauda directions over time. ${ }^{27}$ Further, increased Cho and reduced NAA levels are well documented in various destructive processes with the spinal cord and brain, including multiple sclerosis, spinal cord injury and neoplasm, thus supporting the use of these metabolites as a potential measure of spinal cord viability.

In the present study, we found that a higher Cho/NAA is significantly correlated with a poorer neurological function. This significant inverse relationship between Cho/NAA and mJOA score is a novel and important finding. A number of different conventional
MRI characteristics (for example, spinal cord signal change, spinal cord diameter and so on) have been analyzed in an attempt to find radiographical features that correlate with neurological status in CSM patients, yet none have been able to consistently correlate with clinical condition. If proven reliable with future study, MRS-derived biomarkers such as Cho/NAA could be potentially be utilized clinically in various capacities including the monitoring of asymptomatic or mildly affected patients with advanced cervical degenerative spine disease for progressive spinal cord injury, or as predictors of clinical outcome in patients undergoing surgical intervention.

\section{Study limitations}

Obtaining reliable MR spectroscopic data within the spinal cord can be challenging due to cord or patient movement, difficulty with magnetic field shimming, and partial volume contamination. As such, a potential limitation is lack of reliability data of obtaining MR spectra within the spinal cord. Additionally, placement of the excitation voxel was standardized to the C2 spinal level; however, metabolite concentrations and ratios measured in each patient are likely to be dependent on both the severity of myelopathy and the distance from the site of measurement to the level of injury. Future studies are needed to address spinal cord MR spectroscopic reliability, the influence of the distance between excitation volume position and site of injury on MRS metabolites, and MR spectroscopic changes in patients with severe degrees of neurological dysfunction.

\section{CONCLUSIONS}

MRS may capture some of the early and late spinal cord cellular biochemical changes that occur in patients with advanced cervical spondylosis and CSM. The Cho/NAA ratio has a significant correlation with the mJOA score, providing a potentially clinical useful radiographical biomarker in the management of cervical spondylosis patients.

\section{DATA ARCHIVING}

There were no data to deposit.

\section{CONFLICT OF INTEREST}

The authors declare no conflict of interest. 
1 Moore AP, Blumhardt LD. A prospective survey of the causes of non-traumatic spastic paraparesis and tetraparesis in 585 patients. Spinal Cord 1997; 35: 361-367.

2 Young WF. Cervical spondylotic myelopathy: a common cause of spinal cord dysfunction in older persons. Am Fam Physician 2000; 62: 1064-1070.

3 Wang MC, Chan L, Maiman DJ, Kreuter W, Deyo RA. Complications and mortality associated with cervical spine surgery for degenerative disease in the United States. Spine 2007; 32: 342-347.

4 Sampath P, Bendebba M, Davis JD, Ducker TB. Outcome of patients treated for cervical myelopathy. A prospective, multicenter study with independent clinical review. Spine 2000; 25: 670-676.

5 Irwin ZN, Hilibrand A, Gustavel M, McLain R, Shaffer W, Myers M et al. Variation in surgical decision making for degenerative spinal disorders. Part II: cervical spine. Spine 2005; 30: 2214-2219.

6 Harkey HL, al-Mefty O, Marawi I, Peeler DF, Haines DE, Alexander LF. Experimental chronic compressive cervical myelopathy: effects of decompression. J Neurosurg 1995; 83: 336-341.

7 Fernandez de Rota JJ, Meschian S, Fernandez de Rota A, Urbano V, Baron M. Cervical spondylotic myelopathy due to chronic compression: the role of signal intensity changes in magnetic resonance images. J Neurosurg Spine 2007; 6: 17-22.

8 Yukawa Y, Kato F, Ito K, Horie Y, Hida T, Machino M et al. Postoperative changes in spinal cord signal intensity in patients with cervical compression myelopathy: comparison between preoperative and postoperative magnetic resonance images. J Neurosurg Spine 2008; 8: 524-528.

9 Yukawa $\mathrm{Y}$, Kato F, Yoshihara H, Yanase M, Ito K. MR T2 image classification in cervical compression myelopathy: predictor of surgical outcomes. Spine 2007; 32 : 1675-1678; discussion 1679.

10 Holly LT, Freitas B, McArthur DL, Salamon N. Proton magnetic resonance spectroscopy to evaluate spinal cord axonal injury in cervical spondylotic myelopathy. J Neurosurg Spine 2009; 10: 194-200.

11 Yonenobu K, Abumi K, Nagata K, Taketomi E, Ueyama K. Interobserver and intraobserver reliability of the japanese orthopaedic association scoring system for evaluation of cervical compression myelopathy. Spine (Phila Pa 1976) 2001; 26 : 1890-1894; discussion 1895

12 Benzel EC, Lancon J, Kesterson L, Hadden T. Cervical laminectomy and dentate ligament section for cervical spondylotic myelopathy. J Spinal Disord 1991; 4: 286-295.

13 Provencher SW. Estimation of metabolite concentrations from localized in vivo proton NMR spectra. Magn Reson Med 1993; 30: 672-679.
14 Provencher SW. Automatic quantitation of localized in vivo $1 \mathrm{H}$ spectra with LCModel. NMR Biomed 2001; 14: 260-264.

15 Tierney RT, Maldjian C, Mattacola CG, Straub SJ, Sitler MR. Cervical Spine Stenosis Measures in Normal Subjects. J Athl Train 2002; 37: 190-193.

16 Torg JS, Corcoran TA, Thibault LE, Pavlov H, Sennett BJ, Naranja RJ Jr et al. Cervical cord neurapraxia: classification, pathomechanics, morbidity, and management guidelines. J Neurosurg 1997; 87: 843-850.

17 Herzog RJ, Wiens JJ, Dillingham MF, Sontag MJ. Normal cervical spine morphometry and cervical spinal stenosis in asymptomatic professional football players. Plain film radiography, multiplanar computed tomography, and magnetic resonance imaging. Spine (Phila Pa 1976) 1991; 16 (6 Suppl), S178-S186.

18 Elliott JM, Pedler AR, Cowin G, Sterling M, McMahon K. Spinal cord metabolism and muscle water diffusion in whiplash. Spinal Cord 2011; 50: 474-476.

19 Ciccarelli O, Wheeler-Kingshott CA, McLean MA, Cercignani M, Wimpey K, Miller DH et al. Spinal cord spectroscopy and diffusion-based tractography to assess acute disability in multiple sclerosis. Brain 2007; 130 (Pt 8), 2220-2231.

20 Marliani AF, Clementi V, Albini Riccioli L, Agati R, Carpenzano M, Salvi F et al. Quantitative cervical spinal cord 3T proton MR spectroscopy in multiple sclerosis. AJNR Am J Neuroradiol 2010; 31: 180-184.

21 Verma P, Garcia-Alias G, Fawcett JW. Spinal cord repair: bridging the divide. Neurorehabil Neural Repair 2008; 22: 429-437.

22 Erschbamer M, Oberg J, Westman E, Sitnikov R, Olson L, Spenger C. 1H-MRS in spinal cord injury: acute and chronic metabolite alterations in rat brain and lumbar spinal cord. Eur J Neurosci 2011; 33: 678-688.

23 Ashwal S, Holshouser B, Tong K, Serna T, Osterdock R, Gross M et al. Proton MR spectroscopy detected glutamate/glutamine is increased in children with traumatic brain injury. J Neurotrauma 2004; 21: 1539-1552.

24 Blamire AM, Cader S, Lee M, Palace J, Matthews PM. Axonal damage in the spinal cord of multiple sclerosis patients detected by magnetic resonance spectroscopy. Magn Reson Med 2007; 58: 880-885.

25 Moffett JR, Ross B, Arun P, Madhavarao CN, Namboodiri AM. N-Acetylaspartate in the CNS: from neurodiagnostics to neurobiology. Prog Neurobiol 2007; 81: 89-131.

26 Sajja BR, Wolinsky JS, Narayana PA. Proton magnetic resonance spectroscopy in multiple sclerosis. Neuroimaging Clin N Am 2009; 19: 45-58.

27 Ellingson BM, Schmit BD, Kurpad SN. Lesion growth and degeneration patterns measured using diffusion tensor 9.4-T magnetic resonance imaging in rat spinal cord injury. J Neurosurg Spine 2010; 13: 181-192. 